\title{
Dynamic inventory of moths of Savitribai Phule Pune University (Pune, India) through crowdsourcing via iNaturalist.
}

\section{Bhalchandra Pujari1}

Department of scientific computing modeling and simulation, Savitribai Phule Pune University, Ganeshkhind, Pune 411007

${ }^{1}$ Address for correspondence: bspujari@scms.unipune.ac.in 


\section{Abstract}

We present here the checklist of moths (Lepidoptera: Heterocera) for the campus of Savitribai Phule Pune University, situated in the metropolis of Pune in the state Maharashra in India. We report identification of 189 unique genera along with 154 unique species. Despite the relative small size of the observation area and the location being at the heart of a busy metropolis, the moths were found to be of diverse variety, with 26 different families and 76 tribes.

The identifications of the species was crowd-sourced via iNaturalist.org. An automated program was developed to fetch the identification and generate the list. The program is also being made available open source. As a result the checklist remains relevant all the time with newer identification, correcting existing identification (if required) and taxonomic updates of the future. 


\section{Introduction}

The bustling metropolis of Pune is located at the western part of India at $18^{\circ} 31^{\prime} 13^{\prime \prime} \mathrm{N}$ and $73^{\circ} 51^{\prime} 24^{\prime \prime} \mathrm{E}$ (Fig 1(a)). The metropolitan area of the city has estimated population of 7.4 million (Wikipedia (2021)) and is home to several education institutes including Savitribai Phule Pune University (formerly known as University of Pune). The city is located at the foothills of Western Ghats (Sahyadri mountain ranges) and is rich in biodiversity.

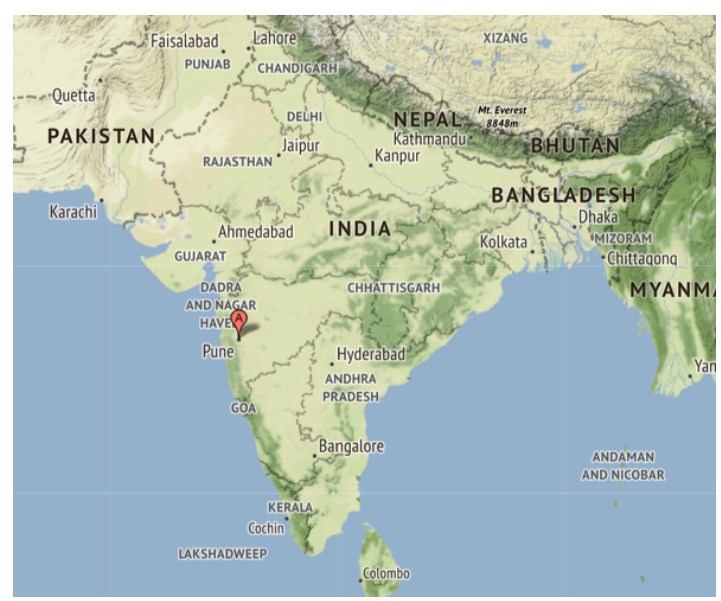

(a)

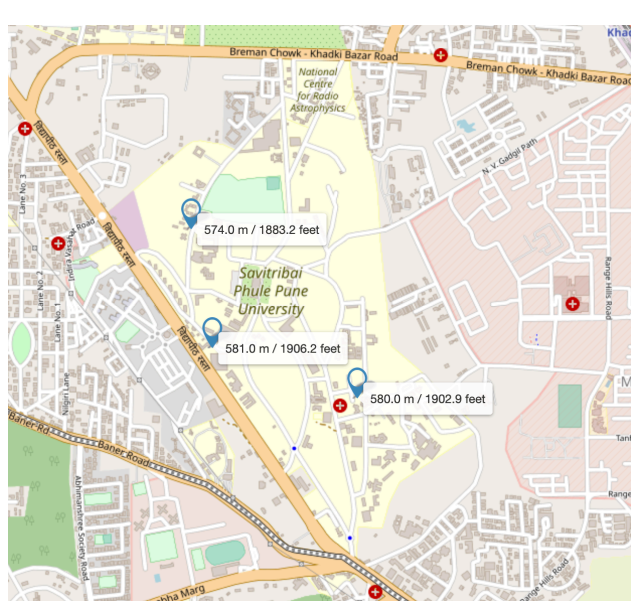

(b)

Fig 1: (a) Pune city, shown with red marker, is located at the western part of India in the state of Maharashtra. It lies west of the Western Ghats. (b) Map of the university (shaded in yellow) along with the locations (in blue) where most of the observations were carried out. Courtesy: ACME mapper/Openstreetmap.

Established in 1949 the university is spread across the area of 411 acres and is considered to be a prime institute in India. The campus is located roughly at the elevation of $580 \mathrm{~m}$ from mean sea level. Ecologically one the unique features of the university campus is the presence of large numbers of Dalbergia melanoxylon (African blackwood) trees, believed to have been introduced in colonial times by British government. Such a large population of these trees is not reported anywhere in India. Apart from Dalbergia 
melanoxylon there are a handful of introduced species like Couroupita guianensis (cannonball), guazuma ulmifolia (West Indian elm), Libidibia coriaria (divi-divi), adansonia (baobabs) among others. The campus also hosts multiple native trees mainly ficus benghalensis (Indian banyan), ficus religiosa (sacred fig), Cassia fistula (golden rain), jacaranda mimosifolia (blue jacaranda) etc.

Despite the rich biodiversity we could not find the comprehensive checklist for the campus or even broader metropolis region. One of the most important works in the nearby region on lepidoptera inventory is due to Shubhalaxmi et. al. (2011) wherein the authors have explored the said fauna in Western Ghats. Their areas of observations has been mainly the hilly region with dense forest cover. They have reported a rich fauna but it is expected that an urban environment is unlikely to support same diversity. The other records do exist from northern Maharashtra (Gurule and Nikam $(2011,2013)$, but the terrain and the climate is significantly differ from that in Pune. Therefore it was imperative to document the Lepidoptera fauna in its current state for the university campus.

\section{Methodology}

To generate the checklist we decided to utilise the power of crowd sourcing using modern technologies. With its powerful Artificial Intelligence engine as well as presence of various taxonomists, iNaturalist (iNaturalist(2021)) was our choice of technology. iNaturalist is a platform for scientists and citizens alike to share the observations from all across the globe. The records are available to public and are naturally peer-reviewed at all the times. Additionally the records from iNaturalist is also shared with Global Biodiversity Information Facility (GBIF(2021)) for broader scientific use.

For data collections we carried more than two years of observations from 2019 to 2021 and ongoing. We periodically observed the walls lit with $60 \mathrm{~W}$ compact fluorescent lamps located mainly at three locations on the campus: 1) near Jaykar library, 2) Faculty house 
and 3) department of scientific computing modeling \& simulation. Some insignificant number of observations were also made at other location across the campus. To be the noninvasive, no separate traps were set up; neither any moth was captured for the observation. As a result some of the identification had to be limited to genera level.

\section{Results}

Before we start discussing the findings from the observation it is worth pointing out that results we are about to present are dynamic in nature. That means over the period of time some updates are expected due to addition of new observation, correction of existing identification or taxonomic changes of future. This ensures it will be continually relevant and never be outdated. In order to stay up-to-date the dynamic version of the checklist is also made available on https://gitlab.com/bspujari/sppu moths. Additionally an interactive webpage is also maintained at author's webpge ${ }^{2}$. Moreover for sake of accessibility the complete Python program that fetches the data from iNaturalist and compiles the list, is made available - open source ${ }^{3}$ - on both of these pages.

At the time of writing of this manuscript, total of 880 observations were made with 737 identification at various level of taxonomy. The list of uniquely identified species is collected in Table 1, at the end of this section. The table also show an "Obs ID" with each observations which is a unique identification number assigned by iNaturalist. One can simply use inaturalist.org/observations/<<Obs ID >> to reach the dedicated observation page which includes the details of taxa as well as media associated with the observation. Additionally any taxonomic updates are also reflected on the page, for example, inaturalist.org/observations/30996315 shows how Pyrausta panopealis was

\footnotetext{
2 http://cms.unipune.ac.in/ bspujari/SPPU_Lepidoptera

${ }^{3}$ GNU Afferro General Public License (GPL) V-3
} 
swapped to Pyrausta phoenicealis, with the reasoning given on inaturalist.org/ taxon changes/64986.

These observations in total represent 26 families as shown in Fig 2, with five most abundant families being Erebidae (234), Geometridae (147), Crambidae (117), Noctuidae (86) and Pyralidae (23), where the bracketed quantities indicate the observation count. It is worth contrasting the reports to that of Shubhalaxmi et al (Shubhalaxmi (2011)) . Both Erebidae, Geometridae families are most abundant in their observations too, however their third abundant family Sphingidae - with 45 species of their 418 total - is considerably absent with merely 13 observations of 7 species.Similarly in the campus of Goa university (Goa, India) only 9 species of Sphingidae have been reported (Gurule and Brookes (2021)) . It is thus reasonable to assume that the urban environment does not suit the host plants required for the Sphingidae.

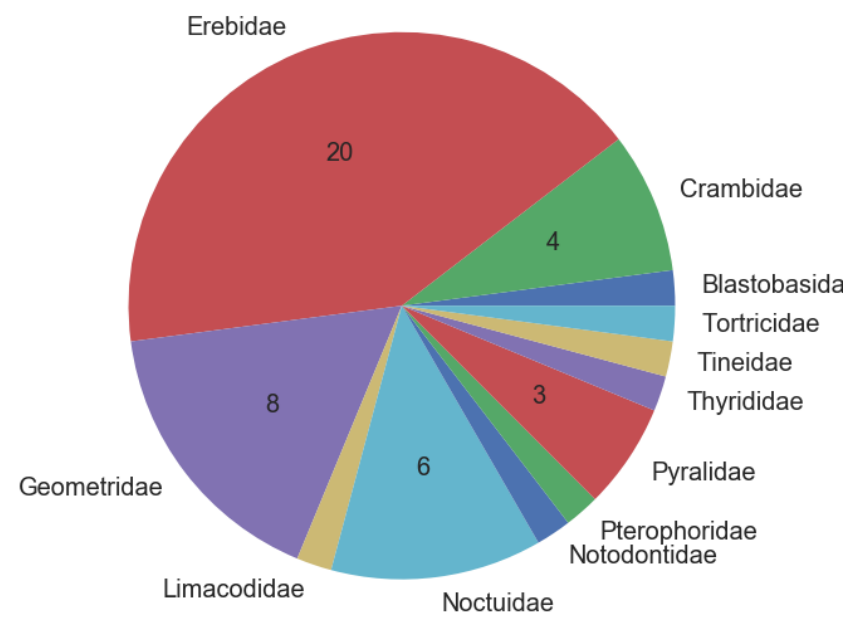

Fig 2: Distribution of families in observed. The numbers indicate uniquely identification of species. Count smaller than 2 are not shown for sake of clarity.

On the other hand, there are some interesting species seen that are rarely reported in Pune or even in India. For example: Spatalia argentifera (Obs ID: 32642126), Sauris 
eupitheciata (Obs Id: 33435892) , Idaea costiguttata (Obs Id: 35704086), Ascotis selenaria (Obs Id: 63959768), Entomogramma torsa (Obs Id: 34574134), Hulodes drylla (Obs Id: 38708649), Hyposada hydrocampata (Obs Id: 33289004). Reports of such species are rare in India and it is indeed hearting to see their presence on the campus. The other notable species is Sommeria marchalii. At the time of preparing this manuscript 49 of 54 observations from India are from Maharashtra state, specifically from the narrow region of western ghats. Pune being in the same region, we have total of eight observations of the species. It appears that this species is fairly localised in west and south of India. Arsacia rectalis is also fairly abundant on the campus. It is reported that the larvae of the species feeds on the plants of Dalbergia genus. It can be speculated - but remains to be verified that they also feed on Dalbergia melanoxylon which as mentioned above is very common on the campus.

Below in Table 1, we present the detailed checklist. As suggested before, the list is expected to grow, modify over the period of time and thus we associate a date with it. What is being released here should be considered as a snapshot at the time of release of this document, and for all future purposes readers are encouraged to check the URLs given before. 


\begin{tabular}{|l|l|l|l|l|r|}
\hline & Family & Subfamily & Genus & Species & Obs ID \\
\hline 1 & Blastobasidae & Blastobasinae & & & 83239194 \\
\hline 2 & Bombycidae & Bombycinae & Trilocha & $\begin{array}{l}\text { Trilocha } \\
\text { varians }\end{array}$ & 35221517 \\
\hline 3 & Bombycidae & Bombycinae & Trilocha & & 63140525 \\
\hline 4 & Choreutidae & Choreutinae & & & 35933515 \\
\hline 5 & $\begin{array}{l}\text { Cosmopterigida } \\
\text { e }\end{array}$ & Cosmopteriginae & Pyroderces & & 31478222 \\
\hline 6 & Crambidae & Acentropinae & Elophila & & 32675514 \\
\hline 7 & Crambidae & Acentropinae & Eoophyla & $\begin{array}{l}\text { Eoophyla } \\
\text { sejunctalis }\end{array}$ & 36080054 \\
\hline 8 & Crambidae & Acentropinae & Parapoynx & $\begin{array}{l}\text { Parapoynx } \\
\text { stagnalis }\end{array}$ & 62869586 \\
\hline 9 & Crambidae & Acentropinae & & & 35642095 \\
\hline 10 & Crambidae & Crambinae & Culladia & & 55300272 \\
\hline 11 & Crambidae & Crambinae & & & 85166676 \\
\hline 12 & Crambidae & Crambinae & Glaucocharis & & 33410558 \\
\hline 13 & Crambidae & Cybalomiinae & Hendecasis & $\begin{array}{l}\text { Hendecasis } \\
\text { duplifascialis }\end{array}$ & 31476720 \\
\hline 14 & Crambidae & Glaphyriinae & Noorda & & 59012406 \\
\hline 15 & Crambidae & Lathrotelinae & Sufetula & & 53369436 \\
\hline 16 & Crambidae & Pyraustinae & Euclasta & & 53598311 \\
\hline 17 & Crambidae & Pyraustinae & Pyrausta & Pyrausta \\
phoenicealis & 53752820 \\
\hline 18 & Crambidae & Pyraustinae & Pyrausta & & 33247089 \\
\hline 19 & Crambidae & Pyraustinae & & & \\
\hline & & & & & \\
\hline
\end{tabular}




\begin{tabular}{|c|c|c|c|c|c|}
\hline 20 & Crambidae & Pyraustinae & Paliga & $\begin{array}{l}\text { Paliga } \\
\text { machoeralis }\end{array}$ & 35328164 \\
\hline 21 & Crambidae & Spilomelinae & Agrotera & & 35703633 \\
\hline 22 & Crambidae & Spilomelinae & Haritalodes & $\begin{array}{l}\text { Haritalodes } \\
\text { derogata }\end{array}$ & 61877039 \\
\hline 23 & Crambidae & Spilomelinae & Lygropia & $\begin{array}{l}\text { Lygropia } \\
\text { distorta }\end{array}$ & 32280175 \\
\hline 24 & Crambidae & Spilomelinae & Nosophora & $\begin{array}{l}\text { Nosophora } \\
\text { euryterminalis }\end{array}$ & 30612736 \\
\hline 25 & Crambidae & Spilomelinae & $\begin{array}{l}\text { Eurrhyparod } \\
\text { es }\end{array}$ & $\begin{array}{l}\text { Eurrhyparodes } \\
\text { bracteolalis }\end{array}$ & 33565981 \\
\hline 26 & Crambidae & Spilomelinae & $\begin{array}{l}\text { Eurrhyparod } \\
\text { es }\end{array}$ & & 52688564 \\
\hline 27 & Crambidae & Spilomelinae & $\begin{array}{l}\text { Herpetogram } \\
\text { ma }\end{array}$ & $\begin{array}{l}\text { Herpetogramm } \\
\text { a licarsisalis }\end{array}$ & 84010982 \\
\hline 28 & Crambidae & Spilomelinae & $\begin{array}{l}\text { Herpetogram } \\
\text { ma }\end{array}$ & & 53959407 \\
\hline 29 & Crambidae & Spilomelinae & Hydriris & $\begin{array}{l}\text { Hydriris } \\
\text { ornatalis }\end{array}$ & 36093562 \\
\hline 30 & Crambidae & Spilomelinae & Lamprosema & $\begin{array}{l}\text { Lamprosema } \\
\text { commixta }\end{array}$ & 31760798 \\
\hline 31 & Crambidae & Spilomelinae & Hymenia & $\begin{array}{l}\text { Hymenia } \\
\text { perspectalis }\end{array}$ & 35221259 \\
\hline 32 & Crambidae & Spilomelinae & Spoladea & $\begin{array}{l}\text { Spoladea } \\
\text { recurvalis }\end{array}$ & 64183228 \\
\hline 33 & Crambidae & Spilomelinae & & & 34731071 \\
\hline 34 & Crambidae & Spilomelinae & Botyodes & & 37126765 \\
\hline 35 & Crambidae & Spilomelinae & Cirrhochrista & $\begin{array}{l}\text { Cirrhochrista } \\
\text { brizoalis }\end{array}$ & 31886094 \\
\hline
\end{tabular}




\begin{tabular}{|c|c|c|c|c|c|}
\hline 36 & Crambidae & Spilomelinae & Conogethes & $\begin{array}{l}\text { Conogethes } \\
\text { punctiferalis }\end{array}$ & 35397500 \\
\hline 37 & Crambidae & Spilomelinae & Conogethes & & 31848999 \\
\hline 38 & Crambidae & Spilomelinae & Diaphania & $\begin{array}{l}\text { Diaphania } \\
\text { indica }\end{array}$ & 35308955 \\
\hline 39 & Crambidae & Spilomelinae & Endocrossis & $\begin{array}{l}\text { Endocrossis } \\
\text { flavibasalis }\end{array}$ & 35328269 \\
\hline 40 & Crambidae & Spilomelinae & Glyphodes & $\begin{array}{l}\text { Glyphodes } \\
\text { bivitralis }\end{array}$ & 36098153 \\
\hline 41 & Crambidae & Spilomelinae & Glyphodes & $\begin{array}{l}\text { Glyphodes } \\
\text { onychinalis }\end{array}$ & 30346624 \\
\hline 42 & Crambidae & Spilomelinae & Maruca & Maruca vitrata & 32631608 \\
\hline 43 & Crambidae & Spilomelinae & Maruca & & 33379170 \\
\hline 44 & Crambidae & Spilomelinae & Omiodes & $\begin{array}{l}\text { Omiodes } \\
\text { indicata }\end{array}$ & 35182888 \\
\hline 45 & Crambidae & Spilomelinae & Pachynoa & & 34731070 \\
\hline 46 & Crambidae & Spilomelinae & Poliobotys & $\begin{array}{l}\text { Poliobotys } \\
\text { ablactalis }\end{array}$ & 36908970 \\
\hline 47 & Crambidae & Spilomelinae & Synclera & $\begin{array}{l}\text { Synclera } \\
\text { traducalis }\end{array}$ & 35182822 \\
\hline 48 & Crambidae & Spilomelinae & Synclera & & 36098877 \\
\hline 49 & Crambidae & Spilomelinae & Talanga & $\begin{array}{l}\text { Talanga } \\
\text { sexpunctalis }\end{array}$ & 36098075 \\
\hline 50 & Crambidae & Spilomelinae & Tyspanodes & $\begin{array}{l}\text { Tyspanodes } \\
\text { linealis }\end{array}$ & 31482841 \\
\hline 51 & Crambidae & Spilomelinae & Sameodes & $\begin{array}{l}\text { Sameodes } \\
\text { cancellalis }\end{array}$ & 58577362 \\
\hline 52 & Crambidae & Spilomelinae & Sameodes & & 58209423 \\
\hline 53 & Crambidae & Spilomelinae & & & 53737568 \\
\hline
\end{tabular}




\begin{tabular}{|c|c|c|c|c|c|}
\hline 54 & Crambidae & Spilomelinae & $\begin{array}{l}\text { Cnaphalocro } \\
\text { cis }\end{array}$ & $\begin{array}{l}\text { Cnaphalocrocis } \\
\text { medinalis }\end{array}$ & 35241633 \\
\hline 55 & Crambidae & Spilomelinae & $\begin{array}{l}\text { Cnaphalocro } \\
\text { cis }\end{array}$ & $\begin{array}{l}\text { Cnaphalocrocis } \\
\text { poeyalis }\end{array}$ & 62869599 \\
\hline 56 & Crambidae & Spilomelinae & $\begin{array}{l}\text { Cnaphalocro } \\
\text { cis }\end{array}$ & & 32280688 \\
\hline 57 & Crambidae & Spilomelinae & Bradina & & 35919100 \\
\hline 58 & Crambidae & Spilomelinae & Chabula & $\begin{array}{l}\text { Chabula } \\
\text { acamasalis }\end{array}$ & 30346843 \\
\hline 59 & Crambidae & Spilomelinae & Nausinoe & $\begin{array}{l}\text { Nausinoe } \\
\text { geometralis }\end{array}$ & 65317514 \\
\hline 60 & Crambidae & Spilomelinae & Rehimena & & 31409191 \\
\hline 61 & Crambidae & Spilomelinae & & & 53429912 \\
\hline 62 & Crambidae & & & & 64915239 \\
\hline 63 & Erebidae & Aganainae & Asota & Asota producta & 37780881 \\
\hline 64 & Erebidae & Aganainae & Sommeria & $\begin{array}{l}\text { Sommeria } \\
\text { marchalii }\end{array}$ & 84127270 \\
\hline 65 & Erebidae & Anobinae & Plecoptera & $\begin{array}{l}\text { Plecoptera } \\
\text { quaesita }\end{array}$ & 35328272 \\
\hline 66 & Erebidae & Arctiinae & Creatonotos & $\begin{array}{l}\text { Creatonotos } \\
\text { gangis }\end{array}$ & 36465870 \\
\hline 67 & Erebidae & Arctiinae & Creatonotos & $\begin{array}{l}\text { Creatonotos } \\
\text { transiens }\end{array}$ & 36825350 \\
\hline 68 & Erebidae & Arctiinae & Creatonotos & & 68106291 \\
\hline 69 & Erebidae & Arctiinae & Nyctemera & $\begin{array}{l}\text { Nyctemera } \\
\text { lacticinia }\end{array}$ & 36932618 \\
\hline 70 & Erebidae & Arctiinae & Olepa & Olepa ricini & 29286773 \\
\hline 71 & Erebidae & Arctiinae & Olepa & & 84641908 \\
\hline
\end{tabular}




\begin{tabular}{|l|l|l|l|l|l|}
\hline 72 & Erebidae & Arctiinae & & & 64915250 \\
\hline 73 & Erebidae & Arctiinae & Aemene & & 62869421 \\
\hline 74 & Erebidae & Arctiinae & Brunia & Brunia antica & 64366593 \\
\hline 75 & Erebidae & Arctiinae & Brunia & & 63738900 \\
\hline 76 & Erebidae & Arctiinae & Cyana & Cyana puella & 64087831 \\
\hline 77 & Erebidae & Arctiinae & Miltochrista & & 35348583 \\
\hline 78 & Erebidae & Arctiinae & Nepita & Nepita conferta & 31587642 \\
\hline 79 & Erebidae & Arctiinae & Schistophlep & & 36118272 \\
\hline 80 & Erebidae & Arctiinae & & & \\
\hline 81 & Erebidae & Arctiinae & Syntomoides & $\begin{array}{l}\text { Syntomoides } \\
\text { imaon }\end{array}$ & 69136347 \\
\hline 82 & Erebidae & Arctiinae & & & 63362395 \\
\hline 83 & Erebidae & Arctiinae & & & 63362717 \\
\hline 84 & Erebidae & Boletobiinae & Araeopteron & & 35261406 \\
\hline 85 & Erebidae & Boletobiinae & Ataboruza & $\begin{array}{l}\text { Ataboruza } \\
\text { divisa }\end{array}$ & 35398544 \\
\hline 86 & Erebidae & Boletobiinae & Enispa & Enispa elataria & 31732773 \\
\hline 87 & Erebidae & Boletobiinae & Enispa & Enispa rosellus & 56180047 \\
\hline 88 & Erebidae & Boletobiinae & Enispa & & 56430920 \\
\hline 89 & Erebidae & Boletobiinae & Zurobata & $\begin{array}{l}\text { Zurobata } \\
\text { vacillans }\end{array}$ & 58150851 \\
\hline 90 & Erebidae & Boletobiinae & Cerynea & & 66420871 \\
\hline 91 & Erebidae & Boletobiinae & Eublemma & $\begin{array}{l}\text { Eublemma } \\
\text { abrupta }\end{array}$ & 38025654 \\
\hline 92 & Erebidae & Boletobiinae & Eublemma & $\begin{array}{l}\text { Eublemma } \\
\text { accedens }\end{array}$ & 35133573 \\
\hline
\end{tabular}




\begin{tabular}{|c|c|c|c|c|c|}
\hline 93 & Erebidae & Boletobiinae & Eublemma & $\begin{array}{l}\text { Eublemma } \\
\text { baccalix }\end{array}$ & 30667613 \\
\hline 94 & Erebidae & Boletobiinae & Hyposada & $\begin{array}{l}\text { Hyposada } \\
\text { hydrocampata }\end{array}$ & 33289004 \\
\hline 95 & Erebidae & Boletobiinae & Gesonia & & 34763754 \\
\hline 96 & Erebidae & Boletobiinae & Rhesala & & 31590079 \\
\hline 97 & Erebidae & Boletobiinae & Hypenagonia & & 68366615 \\
\hline 98 & Erebidae & Boletobiinae & & & 59012152 \\
\hline 99 & Erebidae & Calpinae & Eudocima & $\begin{array}{l}\text { Eudocima } \\
\text { homaena }\end{array}$ & 37700285 \\
\hline 100 & Erebidae & Calpinae & Eudocima & $\begin{array}{l}\text { Eudocima } \\
\text { materna }\end{array}$ & 84267002 \\
\hline 101 & Erebidae & Calpinae & Eudocima & $\begin{array}{l}\text { Eudocima } \\
\text { phalonia }\end{array}$ & 36435311 \\
\hline 102 & Erebidae & Erebinae & Acantholipes & $\begin{array}{l}\text { Acantholipes } \\
\text { trajecta }\end{array}$ & 37781003 \\
\hline 103 & Erebidae & Erebinae & Acantholipes & & 69136311 \\
\hline 104 & Erebidae & Erebinae & Hamodes & $\begin{array}{l}\text { Hamodes } \\
\text { propitia }\end{array}$ & 35665225 \\
\hline 105 & Erebidae & Erebinae & $\begin{array}{l}\text { Entomogram } \\
\text { ma }\end{array}$ & $\begin{array}{l}\text { Entomogramm } \\
\text { a torsa }\end{array}$ & 34574134 \\
\hline 106 & Erebidae & Erebinae & Ercheia & & 60586231 \\
\hline 107 & Erebidae & Erebinae & Erebus & $\begin{array}{l}\text { Erebus } \\
\text { caprimulgus }\end{array}$ & 51362512 \\
\hline 108 & Erebidae & Erebinae & Erebus & $\begin{array}{l}\text { Erebus } \\
\text { hieroglyphica }\end{array}$ & 36813776 \\
\hline 109 & Erebidae & Erebinae & Mocis & Mocis frugalis & 59548226 \\
\hline 110 & Erebidae & Erebinae & Mocis & Mocis undata & 36492026 \\
\hline
\end{tabular}




\begin{tabular}{|c|c|c|c|c|c|}
\hline 111 & Erebidae & Erebinae & Ericeia & \begin{tabular}{|l} 
Ericeia \\
inangulata
\end{tabular} & 59955527 \\
\hline 112 & Erebidae & Erebinae & Ericeia & & 64915205 \\
\hline 113 & Erebidae & Erebinae & Hulodes & $\begin{array}{l}\text { Hulodes } \\
\text { caranea }\end{array}$ & 36152289 \\
\hline 114 & Erebidae & Erebinae & Hulodes & Hulodes drylla & 38708649 \\
\hline 115 & Erebidae & Erebinae & Hulodes & & 61067845 \\
\hline 116 & Erebidae & Erebinae & Speiredonia & & 31041150 \\
\hline 117 & Erebidae & Erebinae & Thyas & Thyas coronata & 53481563 \\
\hline 118 & Erebidae & Erebinae & Polydesma & $\begin{array}{l}\text { Polydesma } \\
\text { boarmoides }\end{array}$ & 65755506 \\
\hline 119 & Erebidae & Erebinae & Pericyma & $\begin{array}{l}\text { Pericyma } \\
\text { cruegeri }\end{array}$ & 31852601 \\
\hline 120 & Erebidae & Erebinae & Pericyma & & 63362313 \\
\hline 121 & Erebidae & Erebinae & Achaea & Achaea janata & 66367988 \\
\hline 122 & Erebidae & Erebinae & Bastilla & & 35264905 \\
\hline 123 & Erebidae & Erebinae & Dysgonia & Dysgonia algira & 32220806 \\
\hline 124 & Erebidae & Erebinae & Dysgonia & $\begin{array}{l}\text { Dysgonia } \\
\text { stuposa }\end{array}$ & 32025376 \\
\hline 125 & Erebidae & Erebinae & & & 35640458 \\
\hline 126 & Erebidae & Erebinae & $\begin{array}{l}\text { Sphingomor } \\
\text { pha }\end{array}$ & $\begin{array}{l}\text { Sphingomorph } \\
\text { a chlorea }\end{array}$ & 36403148 \\
\hline 127 & Erebidae & Erebinae & Spirama & & 31155714 \\
\hline 128 & Erebidae & Erebinae & & & 82953826 \\
\hline 129 & Erebidae & Eulepidotinae & Anticarsia & $\begin{array}{l}\text { Anticarsia } \\
\text { irrorata }\end{array}$ & 35261384 \\
\hline 130 & Erebidae & Herminiinae & Hipoepa & & 30908886 \\
\hline
\end{tabular}




\begin{tabular}{|c|c|c|c|c|c|}
\hline 131 & Erebidae & Herminiinae & Hydrillodes & $\begin{array}{l}\text { Hydrillodes } \\
\text { lentalis }\end{array}$ & 31357085 \\
\hline 132 & Erebidae & Herminiinae & Hydrillodes & & 84874755 \\
\hline 133 & Erebidae & Herminiinae & Naarda & & 84571546 \\
\hline 134 & Erebidae & Herminiinae & Nodaria & & 36118450 \\
\hline 135 & Erebidae & Herminiinae & Progonia & $\begin{array}{l}\text { Progonia } \\
\text { umbrifera }\end{array}$ & 56032914 \\
\hline 136 & Erebidae & Herminiinae & Progonia & & 35932694 \\
\hline 137 & Erebidae & Herminiinae & Simplicia & & 64235875 \\
\hline 138 & Erebidae & Herminiinae & Zanclognatha & & 69853856 \\
\hline 139 & Erebidae & Herminiinae & & & 57999407 \\
\hline 140 & Erebidae & Hypeninae & Britha & & 60586215 \\
\hline 141 & Erebidae & Hypeninae & Dichromia & $\begin{array}{l}\text { Dichromia } \\
\text { laesalis }\end{array}$ & 32531841 \\
\hline 142 & Erebidae & Hypeninae & Hypena & $\begin{array}{l}\text { Hypena } \\
\text { laceratalis }\end{array}$ & 35397524 \\
\hline 143 & Erebidae & Hypeninae & Hypena & $\begin{array}{l}\text { Hypena } \\
\text { obacerralis }\end{array}$ & 82953962 \\
\hline 144 & Erebidae & Hypeninae & Hypena & & 57019534 \\
\hline 145 & Erebidae & Hypenodinae & Luceria & & 84853671 \\
\hline 146 & Erebidae & Hypenodinae & & & 30995466 \\
\hline 147 & Erebidae & Lymantriinae & Arctornis & & 35308640 \\
\hline 148 & Erebidae & Lymantriinae & Perina & Perina nuda & 36238224 \\
\hline 149 & Erebidae & Lymantriinae & Lymantria & & 33533623 \\
\hline 150 & Erebidae & Lymantriinae & Artaxa & Artaxa guttata & 36823389 \\
\hline 151 & Erebidae & Lymantriinae & Artaxa & & 82953682 \\
\hline
\end{tabular}




\begin{tabular}{|l|l|l|l|l|l|}
\hline 152 & Erebidae & Lymantrinae & Nygmia & Nygmia icilia & 37423854 \\
\hline 153 & Erebidae & Lymantrinae & Orvasca & $\begin{array}{l}\text { Orvasca } \\
\text { subnotata }\end{array}$ & 65755581 \\
\hline 154 & Erebidae & Lymantriinae & Orvasca & & 69136368 \\
\hline 155 & Erebidae & Lymantriinae & Olene & & 33411335 \\
\hline 156 & Erebidae & Lymantrinae & Orgyia & Orgyia postica & 33535062 \\
\hline 157 & Erebidae & Lymantriinae & Orgyia & & 35182794 \\
\hline 158 & Erebidae & Lymantriinae & & & 35607403 \\
\hline 159 & Erebidae & Lymantriinae & & & 31886103 \\
\hline 160 & Erebidae & Pangraptinae & Egnasia & $\begin{array}{l}\text { Egnasia } \\
\text { mesotypa }\end{array}$ & 31357611 \\
\hline 161 & Erebidae & Pangraptinae & Egnasia & & 56515558 \\
\hline 162 & Erebidae & Scoliopteryginae & Anomis & Anomis flava & 37394111 \\
\hline 163 & Erebidae & Scoliopteryginae & Anomis & & 33072159 \\
\hline 164 & Erebidae & Tinoliinae & Calesia & $\begin{array}{l}\text { Calesia } \\
\text { stillifera }\end{array}$ & 31615787 \\
\hline 165 & Erebidae & & Arsacia & Arsacia rectalis & 84437558 \\
\hline 166 & Erebidae & & & & 84437530 \\
\hline 167 & Euteliidae & Euteliinae & Eutelia & Penicillaria & 33388423 \\
\hline 168 & Euteliidae & Euteliinae & Penicillaria & $\begin{array}{l}\text { Lophoptera } \\
\text { squammigera }\end{array}$ & 57019547 \\
\hline 169 & Euteliidae & Stictopterinae & Lophoptera & 35293021 \\
\hline 170 & Gelechiidae & & & & 34694505 \\
\hline 171 & Geometridae & Desmobathrinae & Derambila & Derambila \\
& & & & & 35997 \\
\hline
\end{tabular}




\begin{tabular}{|c|c|c|c|c|c|}
\hline 172 & Geometridae & Desmobathrinae & Derambila & $\begin{array}{l}\text { Derambila } \\
\text { saponaria }\end{array}$ & 30967570 \\
\hline 173 & Geometridae & Ennominae & Biston & $\begin{array}{l}\text { Biston } \\
\text { suppressaria }\end{array}$ & 55871874 \\
\hline 174 & Geometridae & Ennominae & Biston & & 55871849 \\
\hline 175 & Geometridae & Ennominae & Alcis & & 35261540 \\
\hline 176 & Geometridae & Ennominae & Ascotis & $\begin{array}{l}\text { Ascotis } \\
\text { selenaria }\end{array}$ & 63959768 \\
\hline 177 & Geometridae & Ennominae & Cleora & $\begin{array}{l}\text { Cleora } \\
\text { injectaria }\end{array}$ & 85166645 \\
\hline 178 & Geometridae & Ennominae & Cleora & & 35241732 \\
\hline 179 & Geometridae & Ennominae & Ectropis & $\begin{array}{l}\text { Ectropis } \\
\text { bhurmitra }\end{array}$ & 84853587 \\
\hline 180 & Geometridae & Ennominae & Ectropis & & 37183490 \\
\hline 181 & Geometridae & Ennominae & Hypomecis & & 62869457 \\
\hline 182 & Geometridae & Ennominae & Hyposidra & $\begin{array}{l}\text { Hyposidra } \\
\text { talaca }\end{array}$ & 59012370 \\
\hline 183 & Geometridae & Ennominae & Ruttellerona & $\begin{array}{l}\text { Ruttellerona } \\
\text { cessaria }\end{array}$ & 31404311 \\
\hline 184 & Geometridae & Ennominae & & & 36407203 \\
\hline 185 & Geometridae & Ennominae & Astygisa & & 84010939 \\
\hline 186 & Geometridae & Ennominae & Petelia & $\begin{array}{l}\text { Petelia } \\
\text { medardaria }\end{array}$ & 32349740 \\
\hline 187 & Geometridae & Ennominae & Petelia & & 35917990 \\
\hline 188 & Geometridae & Ennominae & & & 36423227 \\
\hline 189 & Geometridae & Ennominae & $\begin{array}{l}\text { Heterostegan } \\
\mathrm{e}\end{array}$ & & 32570380 \\
\hline
\end{tabular}




\begin{tabular}{|c|c|c|c|c|c|}
\hline 190 & Geometridae & Ennominae & Achrosis & $\begin{array}{l}\text { Achrosis } \\
\text { rondelaria }\end{array}$ & 56809923 \\
\hline 191 & Geometridae & Ennominae & Achrosis & & 81454169 \\
\hline 192 & Geometridae & Ennominae & Chiasmia & $\begin{array}{l}\text { Chiasmia } \\
\text { eleonora }\end{array}$ & 35312645 \\
\hline 193 & Geometridae & Ennominae & Chiasmia & $\begin{array}{l}\text { Chiasmia } \\
\text { emersaria }\end{array}$ & 65517198 \\
\hline 194 & Geometridae & Ennominae & Chiasmia & $\begin{array}{l}\text { Chiasmia } \\
\text { fidoniata }\end{array}$ & 32349860 \\
\hline 195 & Geometridae & Ennominae & Chiasmia & Chiasmia nora & 31092897 \\
\hline 196 & Geometridae & Ennominae & Chiasmia & $\begin{array}{l}\text { Chiasmia } \\
\text { pluviata }\end{array}$ & 59011982 \\
\hline 197 & Geometridae & Ennominae & & & 62044205 \\
\hline 198 & Geometridae & Ennominae & Hyperythra & $\begin{array}{l}\text { Hyperythra } \\
\text { lutea }\end{array}$ & 67439764 \\
\hline 199 & Geometridae & Ennominae & & & 56029020 \\
\hline 200 & Geometridae & Geometrinae & Agathia & & 30782803 \\
\hline 201 & Geometridae & Geometrinae & Argyrocosma & $\begin{array}{l}\text { Argyrocosma } \\
\text { inductaria }\end{array}$ & 62182225 \\
\hline 202 & Geometridae & Geometrinae & Comibaena & $\begin{array}{l}\text { Comibaena } \\
\text { cassidara }\end{array}$ & 36147545 \\
\hline 203 & Geometridae & Geometrinae & Comostola & $\begin{array}{l}\text { Comostola } \\
\text { meritaria }\end{array}$ & 33288998 \\
\hline 204 & Geometridae & Geometrinae & Hemithea & $\begin{array}{l}\text { Hemithea } \\
\text { tritonaria }\end{array}$ & 57179702 \\
\hline 205 & Geometridae & Geometrinae & Idiochlora & & 64417968 \\
\hline 206 & Geometridae & Geometrinae & Microloxia & $\begin{array}{l}\text { Microloxia } \\
\text { indecretata } \\
\end{array}$ & 35948320 \\
\hline 207 & Geometridae & Geometrinae & & & 55914366 \\
\hline
\end{tabular}




\begin{tabular}{|c|c|c|c|c|c|}
\hline 208 & Geometridae & Geometrinae & Synchlora & & 29607859 \\
\hline 209 & Geometridae & Geometrinae & & & 84385365 \\
\hline 210 & Geometridae & Larentiinae & Eupithecia & & 84127335 \\
\hline 211 & Geometridae & Larentiinae & Gymnoscelis & & 35948201 \\
\hline 212 & Geometridae & Larentiinae & & & 35949740 \\
\hline 213 & Geometridae & Larentiinae & Sauris & $\begin{array}{l}\text { Sauris } \\
\text { eupitheciata }\end{array}$ & 33435892 \\
\hline 214 & Geometridae & Larentiinae & Sauris & & 32315761 \\
\hline 215 & Geometridae & Sterrhinae & Anisephyra & $\begin{array}{l}\text { Anisephyra } \\
\text { ocularia }\end{array}$ & 84869021 \\
\hline 216 & Geometridae & Sterrhinae & $\begin{array}{l}\text { Chrysocrasp } \\
\text { eda }\end{array}$ & $\begin{array}{l}\text { Chrysocrasped } \\
\text { a faganaria }\end{array}$ & 35331122 \\
\hline 217 & Geometridae & Sterrhinae & $\begin{array}{l}\text { Chrysocrasp } \\
\text { eda }\end{array}$ & $\begin{array}{l}\text { Chrysocrasped } \\
\text { a olearia }\end{array}$ & 31840417 \\
\hline 218 & Geometridae & Sterrhinae & $\begin{array}{l}\text { Chrysocrasp } \\
\text { eda }\end{array}$ & $\begin{array}{l}\text { Chrysocrasped } \\
\text { a sanguinea }\end{array}$ & 30781461 \\
\hline 219 & Geometridae & Sterrhinae & $\begin{array}{l}\text { Chrysocrasp } \\
\text { eda }\end{array}$ & & 84874711 \\
\hline 220 & Geometridae & Sterrhinae & Cyclophora & & 33410592 \\
\hline 221 & Geometridae & Sterrhinae & & & 54274591 \\
\hline 222 & Geometridae & Sterrhinae & Traminda & $\begin{array}{l}\text { Traminda } \\
\text { mundissima }\end{array}$ & 84570949 \\
\hline 223 & Geometridae & Sterrhinae & Scopula & & 33535207 \\
\hline 224 & Geometridae & Sterrhinae & Idaea & $\begin{array}{l}\text { Idaea } \\
\text { amplipennis }\end{array}$ & 84280982 \\
\hline 225 & Geometridae & Sterrhinae & Idaea & $\begin{array}{l}\text { Idaea } \\
\text { costiguttata }\end{array}$ & 35704086 \\
\hline
\end{tabular}




\begin{tabular}{|c|c|c|c|c|c|}
\hline 226 & Geometridae & Sterrhinae & Idaea & $\begin{array}{l}\text { Idaea } \\
\text { macrospila }\end{array}$ & 85093135 \\
\hline 227 & Geometridae & Sterrhinae & Idaea & Idaea violacea & 84281139 \\
\hline 228 & Geometridae & Sterrhinae & Idaea & & 85093190 \\
\hline 229 & Geometridae & Sterrhinae & & & 83477220 \\
\hline 230 & Geometridae & & & & 57887290 \\
\hline 231 & Gracillariidae & Phyllocnistinae & Phyllocnistis & $\begin{array}{l}\text { Phyllocnistis } \\
\text { citrella }\end{array}$ & 29286787 \\
\hline 232 & Hyblaeidae & & Hyblaea & Hyblaea puera & 31357389 \\
\hline 233 & Hyblaeidae & & Hyblaea & & 31356697 \\
\hline 234 & Lasiocampidae & Lasiocampinae & Gastropacha & & 36825374 \\
\hline 235 & Lecithoceridae & Lecithocerinae & & & 57999454 \\
\hline 236 & Lecithoceridae & & & & 31518704 \\
\hline 237 & Limacodidae & Limacodinae & Parasa & Parasa lepida & 30971471 \\
\hline 238 & Limacodidae & Limacodinae & Thosea & & 84524856 \\
\hline 239 & Limacodidae & Limacodinae & & & 68444481 \\
\hline 240 & Limacodidae & & & & 37254103 \\
\hline 241 & Noctuidae & Acontiinae & Acontia & Acontia crocata & 30513989 \\
\hline 242 & Noctuidae & Acontiinae & Acontia & & 32475575 \\
\hline 243 & Noctuidae & Aediinae & Aedia & & 35293010 \\
\hline 244 & Noctuidae & Bagisarinae & Amyna & & 56179937 \\
\hline 245 & Noctuidae & Bagisarinae & Xanthodes & $\begin{array}{l}\text { Xanthodes } \\
\text { albago }\end{array}$ & 56129098 \\
\hline 246 & Noctuidae & Condicinae & Condica & Condica illecta & 32587735 \\
\hline 247 & Noctuidae & Condicinae & Condica & & 57614197 \\
\hline
\end{tabular}




\begin{tabular}{|c|c|c|c|c|c|}
\hline 248 & Noctuidae & Eustrotiinae & Maliattha & $\begin{array}{l}\text { Maliattha } \\
\text { quadripartita }\end{array}$ & 57716071 \\
\hline 249 & Noctuidae & Eustrotiinae & Maliattha & $\begin{array}{l}\text { Maliattha } \\
\text { separata }\end{array}$ & 61067936 \\
\hline 250 & Noctuidae & Eustrotiinae & Maliattha & $\begin{array}{l}\text { Maliattha } \\
\text { signifera }\end{array}$ & 85001027 \\
\hline 251 & Noctuidae & Eustrotiinae & Maliattha & & 59548118 \\
\hline 252 & Noctuidae & Eustrotiinae & Ozarba & $\begin{array}{l}\text { Ozarba } \\
\text { punctigera }\end{array}$ & 56029045 \\
\hline 253 & Noctuidae & Eustrotiinae & Ozarba & & 31150988 \\
\hline 254 & Noctuidae & Heliothinae & Helicoverpa & $\begin{array}{l}\text { Helicoverpa } \\
\text { armigera }\end{array}$ & 72422010 \\
\hline 255 & Noctuidae & Heliothinae & Helicoverpa & & 42215447 \\
\hline 256 & Noctuidae & Heliothinae & Heliothis & & 31483173 \\
\hline 257 & Noctuidae & Noctuinae & & & 53429331 \\
\hline 258 & Noctuidae & Noctuinae & Polytela & $\begin{array}{l}\text { Polytela } \\
\text { gloriosae }\end{array}$ & 30971472 \\
\hline 259 & Noctuidae & Noctuinae & Agrotis & & 30295381 \\
\hline 260 & Noctuidae & Noctuinae & & & 81454681 \\
\hline 261 & Noctuidae & Noctuinae & Spodoptera & $\begin{array}{l}\text { Spodoptera } \\
\text { litura }\end{array}$ & 65681062 \\
\hline 262 & Noctuidae & Noctuinae & Spodoptera & & 33320910 \\
\hline 263 & Noctuidae & Noctuinae & & & 66466249 \\
\hline 264 & Noctuidae & Plusiinae & Chrysodeixis & $\begin{array}{l}\text { Chrysodeixis } \\
\text { acuta }\end{array}$ & 35230458 \\
\hline 265 & Noctuidae & Plusiinae & Chrysodeixis & & 58400591 \\
\hline 266 & Noctuidae & Plusiinae & Ctenoplusia & $\begin{array}{l}\text { Ctenoplusia } \\
\text { albostriata }\end{array}$ & 64915105 \\
\hline
\end{tabular}




\begin{tabular}{|c|c|c|c|c|c|}
\hline 267 & Noctuidae & Plusiinae & $\begin{array}{l}\text { Thysanoplusi } \\
\mathrm{a}\end{array}$ & $\begin{array}{l}\text { Thysanoplusia } \\
\text { orichalcea }\end{array}$ & 59011920 \\
\hline 268 & Noctuidae & Plusiinae & Zonoplusia & $\begin{array}{l}\text { Zonoplusia } \\
\text { ochreata }\end{array}$ & 34567555 \\
\hline 269 & Noctuidae & Plusiinae & & & 83806861 \\
\hline 270 & Noctuidae & Plusiinae & & & 35414662 \\
\hline 271 & Noctuidae & & & & 83155117 \\
\hline 272 & Nolidae & Chloephorinae & & & 32280177 \\
\hline 273 & Nolidae & Eariadinae & Earias & & 52688448 \\
\hline 274 & Nolidae & Eligminae & Gadirtha & & 33410661 \\
\hline 275 & Nolidae & Eligminae & Selepa & & 35686599 \\
\hline 276 & Nolidae & Nolinae & Nola & Nola lucidalis & 31886088 \\
\hline 277 & Nolidae & Nolinae & Nola & & 84862171 \\
\hline 278 & Nolidae & Nolinae & Sarbena & & 35932748 \\
\hline 279 & Nolidae & Nolinae & & & 30783149 \\
\hline 280 & Nolidae & $\begin{array}{l}\text { Westermanniina } \\
\text { e }\end{array}$ & $\begin{array}{l}\text { Westermanni } \\
\text { a }\end{array}$ & & 33410603 \\
\hline 281 & Nolidae & & & & 53959245 \\
\hline 282 & Notodontidae & Pygaerinae & Clostera & & 81506804 \\
\hline 283 & Notodontidae & Spataliinae & Spatalia & $\begin{array}{l}\text { Spatalia } \\
\text { argentifera }\end{array}$ & 33234348 \\
\hline 284 & Notodontidae & & & & 29607906 \\
\hline 285 & Oecophoridae & Oecophorinae & Promalactis & & 59548362 \\
\hline 286 & Oecophoridae & & & & 32322017 \\
\hline 287 & Peleopodidae & Acriinae & Acria & & 32315694 \\
\hline 288 & Plutellidae & & Plutella & & 29987203 \\
\hline
\end{tabular}




\begin{tabular}{|l|l|l|l|l|l|}
\hline 289 & Psychidae & & & & 52696758 \\
\hline 290 & Pterophoridae & Pterophorinae & Emmelina & & 35261430 \\
\hline 291 & Pterophoridae & Pterophorinae & & & 34731073 \\
\hline 292 & Pterophoridae & Pterophorinae & Sphenarches & $\begin{array}{l}\text { Sphenarches } \\
\text { anisodactylus }\end{array}$ & 35937678 \\
\hline 293 & Pterophoridae & Pterophorinae & & & 36118427 \\
\hline 294 & Pterophoridae & Pterophorinae & & & 84281026 \\
\hline 295 & Pyralidae & Epipaschiinae & Pococera & & 55300771 \\
\hline 296 & Pyralidae & Phycitinae & Ephestiodes & & 83155201 \\
\hline 297 & Pyralidae & Phycitinae & & & 52696660 \\
\hline 298 & Pyralidae & Phycitinae & & & 65996268 \\
\hline 299 & Pyralidae & Pyralinae & Endotricha & $\begin{array}{l}\text { Endotricha } \\
\text { mesenterialis }\end{array}$ & 33320916 \\
\hline 300 & Pyralidae & Pyralinae & Endotricha & & 84127425 \\
\hline 301 & Pyralidae & Pyralinae & Pyralis & Pyralis pictalis & 53630474 \\
\hline 302 & Pyralidae & & & & 31700197 \\
\hline 303 & Sphingidae & Macroglossinae & Cephonodes & $\begin{array}{l}\text { Cephonodes } \\
\text { hylas }\end{array}$ & 16861802 \\
\hline 304 & Sphingidae & Macroglossinae & Daphnis & Daphnis nerii & 30971479 \\
\hline 305 & Sphingidae & Macroglossinae & Hippotion & $\begin{array}{l}\text { Hippotion } \\
\text { celerio }\end{array}$ & 33200525 \\
\hline 306 & Sphingidae & Macroglossinae & Hippotion & & 35846310 \\
\hline 307 & Sphingidae & Macroglossinae & Theretra & $\begin{array}{l}\text { Theretra } \\
\text { nessus }\end{array}$ & 59195645 \\
\hline 308 & Sphingidae & Macroglossinae & & & 33463418 \\
\hline 309 & Sphingidae & Smerinthinae & Sataspes & & 34566459 \\
\hline
\end{tabular}




\begin{tabular}{|c|c|c|c|c|c|}
\hline 310 & Sphingidae & Sphinginae & Acherontia & Acherontia styx & 33416939 \\
\hline 311 & Sphingidae & Sphinginae & Psilogramma & $\begin{array}{l}\text { Psilogramma } \\
\text { menephron }\end{array}$ & 30971473 \\
\hline 312 & Stathmopodidae & & Stathmopoda & $\begin{array}{l}\text { Stathmopoda } \\
\text { auriferella }\end{array}$ & 53156244 \\
\hline 313 & Thyrididae & Siculodinae & $\begin{array}{l}\text { Hypolampru } \\
\text { s }\end{array}$ & $\begin{array}{l}\text { Hypolamprus } \\
\text { angulalis }\end{array}$ & 83155010 \\
\hline 314 & Thyrididae & Striglininae & Banisia & $\begin{array}{l}\text { Banisia } \\
\text { myrsusalis }\end{array}$ & 31483241 \\
\hline 315 & Thyrididae & & Striglina & $\begin{array}{l}\text { Striglina } \\
\text { scitaria }\end{array}$ & 57291976 \\
\hline 316 & Thyrididae & & & & 35398482 \\
\hline 317 & Tineidae & Erechthiinae & Pyloetis & $\begin{array}{l}\text { Pyloetis } \\
\text { mimosae }\end{array}$ & 65681096 \\
\hline 318 & Tineidae & $\begin{array}{l}\text { Perissomasticina } \\
\text { e }\end{array}$ & Edosa & & 53429242 \\
\hline 319 & Tineidae & Tineinae & Monopis & $\begin{array}{l}\text { Monopis } \\
\text { monachella }\end{array}$ & 55891598 \\
\hline 320 & Tineidae & Tineinae & Phereoeca & & 35360236 \\
\hline 321 & Tortricidae & Olethreutinae & Loboschiza & $\begin{array}{l}\text { Loboschiza } \\
\text { koenigiana }\end{array}$ & 29607704 \\
\hline 322 & Tortricidae & Olethreutinae & & & 33388513 \\
\hline 323 & Tortricidae & Olethreutinae & $\begin{array}{l}\text { Cryptophlebi } \\
\text { a }\end{array}$ & & 35701487 \\
\hline 324 & Tortricidae & Olethreutinae & & & 31343961 \\
\hline 325 & Tortricidae & Olethreutinae & Statherotis & & 33647400 \\
\hline 326 & Tortricidae & Olethreutinae & & & 62869506 \\
\hline 327 & Tortricidae & Olethreutinae & & & 59011616 \\
\hline
\end{tabular}




\begin{tabular}{|l|l|l|l|l|l|}
\hline 328 & Tortricidae & Tortricinae & Clepsis & & 31151101 \\
\hline 329 & Tortricidae & Tortricinae & Homona & & 30971484 \\
\hline 330 & Tortricidae & Tortricinae & & & 57887329 \\
\hline 331 & Tortricidae & Tortricinae & & & 35308960 \\
\hline 332 & Tortricidae & & & & 64087942 \\
\hline 333 & Uraniidae & Epipleminae & Epiplema & $\begin{array}{l}\text { Epiplema } \\
\text { fulvilinea }\end{array}$ & 84452434 \\
\hline 334 & Uraniidae & Epipleminae & Phazaca & & 84571455 \\
\hline 335 & Uraniidae & Microniinae & Micronia & $\begin{array}{l}\text { Micronia } \\
\text { aculeata }\end{array}$ & 59226178 \\
\hline
\end{tabular}

Table 1: List of moths of Savitribai Phule Pune University, retrieved on Jul 01, 2021.

\section{Discussion}

Despite its presence in a busy metropolis the campus of Savitribai Phule Pune University is home to rich Lepidoptera diversity. With a project spanning for more two years we have compiled a checklist of moths and found the presence of 189 genera and 154 species from 26 families. In an attempt to be precise the identification was crowd sourced via inaturalist.org where each identification is effectively peer-reviewed by global community. The identification automatically and periodically retrieved via a Python program - which is also being released with this manuscript. An advantage of such a system is that any addition of new species, or correction to either identification or taxonomic records get automatically incorporated in future versions of checklist. As a result the checklist that is being presented here is dynamic and subsequent changes can be followed up at $\underline{G i t L a b}^{4}$ or

4 https://gitlab.com/bspujari/sppu_moths 
author's webpge 5 . With the given python program anyone should be able to generate similar checklist for area of their interest.

\section{Acknowledgments}

Being a crowd sourced work the author is grateful to all individuals who have help identify the species. In particular author is grateful to Swanand Kesari, Nagabhushan Jyothi(@nagabhushanjyothi),Vijay Barve, Unnikrishanan (@unnikrishanan_mp), Roger Kendrick (@hkmoths), Rohit MG (@rohitmg), Mangesh Kulkarni and Deepashri Saraf. Special thanks are to @po-po-pro for identification as well as critique of the work, and last but most certainly not the least my huge gratitudes are to Piero Toni (@pierotoni10) for all the help and encouragement.

\section{References}

GIBF (2021): Global Biodiversity Information Facility, https://www.gbif.org. [Online; accessed 16-June-2021].

Gurule, S. A and S. M. Nikam (2011): Inventory of lepidopterous insects in North Maharashtra and survey for moth diversity. Flora and Fauna (Jhansi) 17(1), pp. 165-174.

Gurule, S. A and S. M Nikam (2013) : The moths (Lepidoptera: Heterocera) of northern Maharashtra: a preliminary checklist". Journal of Threatened Taxa 5(12). 4693- 4713.

Gurule, S. A. and R. D. Brookes (2021): A preliminary study of moths (Insecta: Lepidoptera) of Goa University Campus, Goa. Rec. zool. Surv. India 121(1), p. 101.

5 http://cms.unipune.ac.in/ bspujari/SPPU_Lepidoptera 
iNaturalist (2021). https://www.inaturalist.org. [Online; accessed 16-June-2021].

Shubhalaxmi, V., R. C. Kendrick, A. Vaidya, N. Kalagi and A. Bhagwat (2011): Inventory of moth fauna (Lepidoptera: Heterocera) of the northern western Ghats, Maharashtra, India" In: Journal of the Bombay Natural History Society 108 (3), p. 183-205.

Wikipedia (2021). Pune - Wikipedia, The Free Encyclopedia. http://en.wikipedia.org/w/ index.php?title=Pune. [Online; accessed 16-June-2021]. 